Voix et Images

voixetimages

\title{
Gabrielle Roy et Margaret Laurence : deux chemins, une recherche de Terrance Hugues
}

\section{Nicole Bourbonnais}

Volume 14, numéro 2 (41), hiver 1989

L’édition littéraire au Québec

URI : https://id.erudit.org/iderudit/200780ar

DOI : https://doi.org/10.7202/200780ar

Aller au sommaire du numéro

Éditeur(s)

Université du Québec à Montréal

ISSN

0318-9201 (imprimé)

1705-933X (numérique)

Découvrir la revue

Citer cet article

Bourbonnais, N. (1989). Gabrielle Roy et Margaret Laurence : deux chemins, une recherche de Terrance Hugues. Voix et Images, 14(2), 330-333.

https://doi.org/10.7202/200780ar d'utilisation que vous pouvez consulter en ligne.

https://apropos.erudit.org/fr/usagers/politique-dutilisation/ 
330 VOIX \& IMAGES / 41, hiver 1989

\section{Gabrielle Roy et Margaret Laurence: deux chemins, une recherche de Terrance Hugues}

par Nicole Bourbonnais, Université d'Ottawa

Chercheurs $d^{\prime}$ horizons ${ }^{1}$ pourrait-on dire des critiques de littérature comparée suivant la belle formule imaginée par Gabrielle Roy pour décrire sa famille voyageuse. Ne cherchent-ils pas eux aussi, fascinés par l'autre et l'ailleurs, à 
repousser les frontières, à nier les séparations, réunissant l'Allemagne et la France, l'Irlande et l'Italie, le Québec et les États-Unis? Comment se fait-il que, dans certains cas, pareille curiosité semble faire défaut? Comment expliquer le nombre relativement restreint d'études comparatives en littérature canadienne et québécoise? Simple indifférence, difficultés posées par les barrières linguistiques, inquiétude quant à l'identité menacée ou tout cela à la fois? Il n'est pas de mon propos ici de procéder à un examen des causes de ce phénomène mais plutôt de constater que, depuis 1970, une plus grande ouverture dans le domaine de la littérature comparée canadienne et québécoise semble se manifester. En témoignent, entre autres, les travaux de R. Sutherland et de P. Stratford ou encore, ce numéro de Voix $\&$ images ${ }^{2}$, consacré à la littérature canadienneanglaise, à l'automne 1984 (dans lequel on trouve, entre autres, le titre des articles et des ouvrages de P. Stratford et de R. Sutherland).

Et voici que nous est donnée une nouvelle preuve de cette ouverture à l'Autre avec la publication d'une étude comparative, consacrée à deux écrivaines d'origine manitobaine, et intitulée Gabrielle Roy et Margaret Laurence: deux chemins, une recherche ${ }^{3}$. Cet ouvrage, issu d'une thèse de doctorat faite à l'université McGill, est l'œuvre de Terrance Hugues, originaire de Vanvouver et auteur de plusieurs traductions. Paru aux Editions du Blé, au beau nom évocateur, ce livre s'inscrit dans une volonté de reconnaissance des écrivains de l'Ouest canadien. On se rappellera la publication, par cette même maison d'édition, de l'étude d'Annette Saint-Pierre consacrée à Gabrielle Roy ainsi que celle d'Hélène Chaput sur Donatien Frémont, journaliste de l'Ouest canadien. Le livre de Hugues s'insère donc dans une double volonté, celle de rendre manifeste la parenté d'esprit des écrivains originaires de l'Ouest canadien et celle de faire avancer la connaissance dans un domaine relativement peu connu.

Après avoir brièvement traité de la problématique de la littérature comparée canadienne et fourni un bref aperçu de l'évolution du roman québécois et canadien, Hugues entreprend de suivre le parcours littéraire des deux romancières manitobaines, tentant de cerner ce qu'il y a de commun dans leur apprentissage et dans les principales étapes de leurs carrières. Il décèle, à juste titre, l'importance de l'éloignement dans le déclenchement du processus créateur et dans l'orientation de la vision sociale. L'auteur consacre la deuxième partie de son ouvrage, la plus substanticlle, à l'étude des personnages communs aux deux auteurcs, soit les personnages féminins, notamment celui de la mère, ainsi que lcs marginaux, tels l'artiste et l'écrivain, l'Esquimau et l'Indien. Enfin, les derniers chapitres, d'ordre plus thématique, traitent de l'espace et des souvenirs d'enfance.

Ccrtes, il faut savoir gré à Terrance Hugues de s'être aventuré dans une contrće inexplorée et confrontant les mondes romanesques de Roy et de Laurence, mais on souhaitcrait que, de ce rapprochement, naissent plus souvent l'inattendu et l'inćdit. Or, rien de bien nouvcau, sinon pour les nćophytes, dans cctic analyse psychologisante des personnages où réapparaissent les licux communs sur le dévouement maternel, le désir d'émancipation, la nostalgie de l'enfance, l'immensité des plaines, le déracinement des étrangers. Où un 
encombrant appareil citationnel et de nombrcuses répétitions -- est-ce un reste de la thèse? - alourdissent inutilement le cheminement de l'analyse. Pourtant, ccrtaines pistes, comme celle justement de l'étranger ou du paria, auraicnt pu dépasser l'inventaire (les étrangers sont partout présents dans Alexandre Chenevert, p. 108) ou le commentaire génćral (les Tonnerre sont souvent méprisés parce qu'ils ne sont ni Blancs ni Indiens, p. 116), pour étudier l'articulation qui leur est donnće au sein de l'ensemble narratif et pour approfondir leur symbolique. Terrance Hugues a parfois d'excellentes intuitions en ce sens:

Les métis sont coupés de leur passé; n'étant ni Blancs ni Cris pur sang, ils sont les êtres partagés par excellence, symbole de l'esprit divisé qui cherche à résoudre l'éternel conflit entre la nature et la culture. (p. 110)

Au lieu des éternelles redites sur l'influence que l'espace exerce sur les personnages (p. 155) - comme si ces personnages étaient des personnes et non de pures fictions -, aurait pu être fort avantageusement développée la notion du «lieu», géographique et mental, comme élément créateur de l'imaginaire des écrivains. À la manière d'un Giono ou d'un Faulkner qui, nourris de lcur terre natale, ont recréé un Sud imaginaire, Margaret Laurence et Gabrielle Roy n'ontelles pas, l'une et l'autre, donné naissance à un Ouest mythique qui structure leur œuvre et agit comme métaphore fondatrice?

Est à déplorer aussi le fait que la création littéraire soit la plupart du temps reléguce aux oubliettes ou réduite à une affaire de compassion ou de sens de l'observation, comme en témoigne la remarque suivante selon laquelle l'originalité de Bonheur d'occasion et de This Side Jordan tient moins à la nouveauté de la forme des récits [...] qu'à leur compassion et à leur réalisme (p. 42). Hugues semble oublier que le réalisme relève d'un choix esthétique conscient, qui exige un travail d'écriture bien spécifique et que toute création littéraire est avant tout une affaire de langage et de formes. Le partage qu'il établit entre les premières œuvres, fondées sur l'expérience observée et les suivantes, fondées sur l'expérience vécue (p. 54) revient encore à faire de l'écriture tantôt une copie conforme des choses, tantôt un simple remaniement de la réalité. En dépit de son affirmation réitćrée sclon laquelle ni l'une ni l'autre des romancières n'a révolutionné le genre romanesque, il y aurait peut-être lieu, au contraire, de s'intéresser aux stratégies discursives de l'une et de l'autre. Margaret Laurence, d'une autre génération que celle de Gabrielle Roy, s'est adonnée à des expériences astucieuses dans le domaine narratif, notamment dans The Diviners où la protagoniste, Morag, raconte sa propre histoire comme si elle était une autre, ce qui crée une distanciation et un dédoublement des plus efficaces. L'attention portée aux techniques narratives aurait permis d'éviter les informations approximatives et imprécises comme celle qui consiste à affirmer d'abord que la narration du roman The Diviners se fait à la troisième personne (p. 53) pour déclarer plus loin que c'est Morag qui assume la narration de sa vie (p. 140).

En dernier lieu, les romans de Margaret Laurence et de Gabrielle Roy, s'ils ont en commun un certain rapport à l'espace et s'ils partagent certaines zones 


\section{CHRONIQUES 333}

d'intérêt, diffèrent pourtant largement tant par la forme que par la perspective. Je songe au thème de la sexualité que chacune aborde dans une lumière qui lui est propre. Et l' «exil» d'une Canadienne française au pays de ses grands-parents peut-il être si proche de l'exil d'une Canadienne anglaise en Afrique? Bref, j'ai l'impression qu'animé d'un fantasme fusionnel, Hugues néglige les écarts et les divergences qui pourraient pourtant être fort révélateurs. Un regard sur l'histoire et la relation entre les deux cultures aurait permis de mieux éclairer les deux univers romanesques aussi bien que de dégager des repères fondamentaux pour l'étude comparée des littératures canadienne et québécoise. Dans un article, consacré au rapport entre les deux littératures, Patricia Smart fait remarquer que les comparatistes d'ici ont eu tendance à négliger les impératifs historiques qui ont défini nos deux cultures et les rapports qui se sont tissés entre elles. ${ }^{4}$

En dépit de nombreuses réserves, je tiens à saluer la publication de Gabrielle Roy et Margaret Laurence: deux chemins, une recherche et à féliciter Terrance Hugues d'avoir ouvert de nouvelles avenues dans le domaine de la littérature comparée canadienne et québécoise, qui en est toujours à ses débuts, voire à ses balbutiements. D'être celui du pionnier et du défricheur, son ouvrage n'en acquiert que plus de valeur et de mérite.

1 Gabrielle Roy, «Mon hćritage du Manitoba», Fragiles Lumières de la terre, Montréal, Stanké, 1982, p. 145.

2 Voix \& images, vol. X, no 1, automne 1984.

3 Terrance Hugues, Gabrielle Roy et Margaret Laurence: deux chemins, une recherche, 2e édition, Saint-Boniface, les Éditions du Blé, 1987 (Solcil).

4 Patricia Smart, «L'espace de nos fictions: quelques réflexions sur nos deux cultures», Voix \& images, op. cit., p. 26. 